\title{
DNA methylation changes of whole blood cells in response to active smoking exposure in adults: a systematic review of DNA methylation studies
}

\author{
Xu Gao ${ }^{1}$, Min Jia', Yan Zhang ${ }^{1}$, Lutz Philipp Breitling ${ }^{1}$ and Hermann Brenner ${ }^{1,2,3^{*}}$
}

\begin{abstract}
Active smoking is a major preventable public health problem and an established critical factor for epigenetic modification. In this systematic review, we identified 17 studies addressing the association of active smoking exposure with methylation modifications in blood DNA, including 14 recent epigenome-wide association studies (EWASs) and 3 gene-specific methylation studies (GSMSs) on the gene regions identified by EWASs. Overall, 1460 smoking-associated CpG sites were identified in the EWASs, of which 62 sites were detected in multiple $(\geq 3)$ studies. The three most frequently reported CpG sites (genes) in whole blood samples were cg05575921 (AHRR), cg03636183 (F2RL3), and cg19859270 (GPR15), followed by other loci within intergenic regions 2q37.1 and 6p21.33. These significant smoking-related genes were further assessed by specific methylation assays in three GSMSs and reflected not only current but also lifetime or long-term exposure to active smoking. In conclusion, this review summarizes the evidences for the use of blood DNA methylation patterns as biomarkers of smoking exposure for research and clinical practice. In particular, it provides a reservoir for constructing a smoking exposure index score which could be used to more precisely quantify long-term smoking exposure and evaluate the risks of smokinginduced diseases.
\end{abstract}

Keywords: DNA methylation, Active smoking, Whole blood cells, Systematic review

\section{Background}

Tobacco smoking is a major public health problem, globally associated with substantial preventable morbidity [1]. In particular, active smoking in adults accounts for a large share of a wide spectrum of chronic diseases, including various forms of cancer, respiratory, and cardiovascular diseases. DNA methylation, one of the main forms of epigenetic modifications, has recently been suggested to play an important role in the pathways of smoking and smoking-induced diseases [2].

Earlier epigenetic studies on active smoking generally applied locus-specific assays to detect cytosine conversion through PCR or DNA sequencing techniques to investigate

\footnotetext{
* Correspondence: h.brenner@dkfz-heidelberg.de

'Division of Clinical Epidemiology and Aging Research, German Cancer Research Center (DKFZ), Im Neuenheimer Feld 581, D-69120 Heidelberg, Germany

${ }^{2}$ Division of Preventive Oncology, German Cancer Research Center (DKFZ) and National Center for Tumor Diseases (NCT), Im Neuenheimer Feld 460, D-69120 Heidelberg, Germany

Full list of author information is available at the end of the article
}

the alteration of genes in tissues and blood samples [3, 4]. These approaches yielded several replicable smokingrelated loci in the candidate genes $M A O A, M A O B$, and COMT, as well as global methylation differences [4]. Following the introduction of Infinium Human Methylation Bead Chip (27 and $450 \mathrm{~K}$ ) assays, numerous smokingrelated CpG sites have been discovered via epigenome-wide association studies (EWASs), which investigated DNA methylation in whole blood samples collected through epidemiological studies. The first such site, cg03636183, located in the gene F2RL3, was reported by Breitling et al. in 2011 [5]. The so far largest EWAS by Zeilinger, reported in 2013, discovered and validated statistically significant differences in the methylation of $187 \mathrm{CpG}$ sites between current smokers and never smokers [6]. Based upon the findings in EWASs, more recent studies focused on blood DNA methylation at specific genes, such as F2RL3 and $A H R R$, to further explore long-term or lifetime correlates of active smoking exposure which might serve as biomarkers for both current and past smoking exposure [7-9].

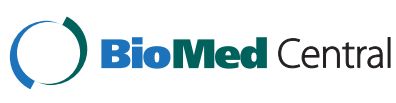

(C) 2015 Gao et al. Open Access This article is distributed under the terms of the Creative Commons Attribution 4.0 International License (http://creativecommons.org/licenses/by/4.0/), which permits unrestricted use, distribution, and reproduction in any medium, provided you give appropriate credit to the original author(s) and the source, provide a link to the Creative Commons license, and indicate if changes were made. The Creative Commons Public Domain Dedication waiver (http://creativecommons.org/publicdomain/zero/1.0/) applies to the data made available in this article, unless otherwise stated. 
In view that blood samples are most commonly available in epidemiological studies and may be particularly relevant for systemic sequelae of smoking and smoking-related health disorders, it is predictable that there will be many EWASs based on whole blood samples in the near future with the spreading application of Illumina assays. Thus, this systematic review aims to summarize the current evidence on the association of active smoking with whole blood DNA methylation among adults, from EWASs and gene-specific studies (GSMSs) on specific gene regions identified by EWASs, to provide applicable suggestions for further smokingrelated methylation studies.

\section{Methods}

We conducted this systematic literature review according to a predefined protocol. Reporting follows the PRISMA statements [10].

\section{Data sources and search strategy}

A systematic literature search was conducted to identify studies assessing smoking-related differential methylation in CpG sites in humans. Databases of PubMed and ISI Web of Science were searched for eligible articles until February 28, 2015. The keyword combinations used in PubMed were as follows: ((("smoking”[MeSH Terms] OR "smoking"[All Fields]) OR ("smoke"[MeSH Terms] OR "smoke"[All Fields])) AND ("methylation" [MeSH Terms] OR "methylation"[All Fields])). The search terms used in ISI Web of Science were as follows: TS $=\left(\left(\left(\right.\right.\right.$ smoking*) ${ }^{*}$ OR (smoke) $)$ AND (methylation) $)$. After filtering the duplicates by Endnote X7, and excluding ineligible articles by title and abstract review, articles with potential relevance for the study topic underwent full-text review.

\section{Study selection}

During title and abstract screening for potentially eligible studies, we used the following exclusion criteria (Fig. 1): 1) not published in English; 2) studies conducted in non-human beings; 3 ) not original articles or articles without full text; 4) no data on smokingmethylation associations; 5) studies addressing global methylation only; 6) studies without any significant results; 7) studies not including adults; and 8) studies not based on blood samples. After a full-text review, therapy-related studies were also excluded, and the remaining articles were finally categorized into EWASs and GSMSs.

\section{Data extraction and quality assessment}

Two investigators (XG, MJ) independently extracted data from the eligible studies. From each study, we summarized available information on first author, country, study design, basic characteristics of samples (including size, gender, and age distribution), type of bio-specimen, leukocyte distribution adjustment (LDA), smoking indicators, number of significant $\mathrm{CpG}$ sites along with their located genes, correction for multiple testing, and estimated effect sizes of smoking exposure on methylation (expressed by $\beta$ values). Effect sizes, a quantitative measure of methylation changes, were calculated between current/former smokers and non-smokers for each CpG site without further discrimination by exposure quantity (effect $\quad$ size $=$ median $/$ mean $\beta_{\text {current } / \text { former }}$ smoker $\quad-$ median/mean $\beta_{\text {never smoker }}$ ).

We assessed the quality of each included article using the following quality criteria: 1) clear definition and description of study population; 2) suitable ascertainment of smoking and covariates (interviews/questionnaires or hospital records); and 3) consideration (through stratification or adjustment) of at least the following covariates in the analysis: age and sex.

Microsoft Excel 2010 (Microsoft Corporation, New Mexico, USA), SAS version 9.3 (SAS Institute Inc., Cary, NC, USA), and Sigmaplot Ver.12.5 (Systat Software Inc. California, USA) were used for data cleaning and to plot the figures, respectively.

\section{Results}

\section{Literature search results}

The initial search yielded 1982 articles using the abovedescribed search terms, 880 from PubMed and 1102 from Web of Science (Fig. 1). Among those, we excluded 786 duplicates and 1178 by title and abstract scan. The remaining 18 studies were selected for a full-text review, of which one therapy-related study was excluded. Crossreferencing revealed no further relevant records. Therefore, 17 articles assessing the association of smoking with blood DNA methylation were eligible for this systematic review, including 14 EWASs $[5,6,11-22]$ and 3 GSMSs [7, 8, 23] which were published between 2011 and 2015 (Tables 1 and 2). Furthermore, we summarized effect sizes of smoking exposure on all reported CpG sites with their corresponding genes (Additional file 1 : Table S1) and plotted loci discovered by multiple $(\geq 3)$ studies with their available effect sizes ( $\beta$ values) in validation panels (i.e., independent replication samples, which should not be affected by the overestimation of true associations that is to be expected in the discovery samples [24] Fig. 2). Additionally, we used the published data of the study by Zeilinger et al. as an example to describe the effect size alterations of multiple discovered loci after smoking cessation (Fig. 3) [6].

\section{Study characteristics and results of EWASs}

All EWASs applied Illumina 27 or $450 \mathrm{~K}$ methylation arrays to uncover smoking-related $\mathrm{CpG}$ sites. Three 


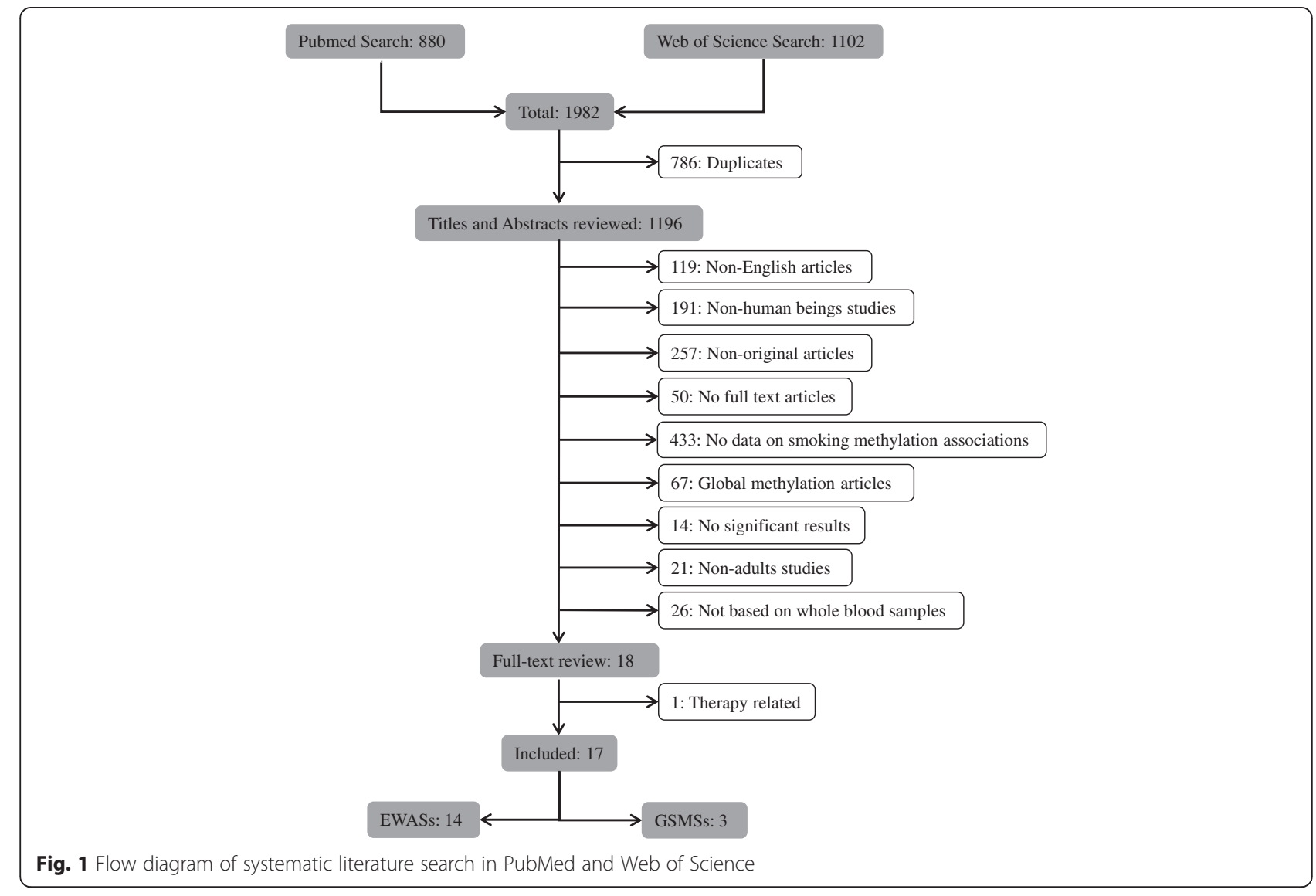

studies carried out pyrosequencing [12, 14, 19], one used MALDI-TOF [5], and one used quantitative-PCR [17] as additional supplementary measurement in their validation phases (Table 1). Six studies were from the USA, seven from Europe $(\mathrm{UK}=4$, Germany $=2$, Sweden $=1)$, and one from Qatar. The population sizes varied from 107 to 1793 in discovery panels, two studies only included males [13, 18], and four only included females $[14,17,19,21]$. Eight studies validated their findings further in internal independent replication samples, while another two studies replicated findings of significant associations from proceeding EWASs [16, 22]. Two studies were carried out in lymphocyte DNA [11, 17], and twelve studies assessed methylation in whole blood DNA. Eight studies adjusted their results for leucocyte distribution $[6,15-18,20-22]$, seven of which used the algorithm of Houseman et al. [25]. However, five studies emphasized that the variability in DNA methylation due to cell composition was probably small and insignificant when compared with the differential methylation patterns that can be observed between smokers and non-smokers $[15-17,20]$. Family-wise error rate (FWER) or false discovery rate (FDR) was applied to adjust multiple testing.

A total of $1460 \mathrm{CpG}$ sites were identified in these EWASs, 62 of them were discovered in multiple $(\geq 3)$ studies. Among the latter, 47 were located in 27 genes (35 were located in the bodies of corresponding genes), and 15 were located in the intergenic regions (Additional file 1: Table S1). Their effect sizes in validation samples are summarized in Additional file 1: Table S1 (range in validation samples, -0.233 (cg05575921) to +0.157 (cg23480 021)), and compared in Fig. 2. The five sites (genes) with the largest effect sizes were cg09935388 (GFI1), cg05575 921 (AHRR), cg23576855 (AHRR), cg06126421 (6p21.33), and cg03636183 (F2RL3). Loci cg03636183 (F2RL3), cg055 75921 (AHRR), and cg19859270 (GPR15) were reported 12, 11 , and 10 times, respectively. Other CpG sites located in intergenic regions of $2 q 37.1$ and $6 p 21.33$ were reported at least four times as well (Additional file 1: Table S1). For all but two sites within gene MYO1G (cg12803068 and cg22132788), smoking was associated with decreased methylation. Eleven loci discovered in multiple $(\geq 3)$ studies were located in $A H R R$, followed by six loci in 2q37.1, four loci in C14orf43, and three loci in GFI1 (Fig. 2).

Figure 3 provides a comparison of effect estimates for these same CpG sites for current and former smokers from the largest EWAS up to date by Zeilinger et al. [6]. A consistent pattern of effects among former smokers that are in the same direction but much smaller than effects among current smokers suggests the reversibility 
Table 1 Characteristics summary of EWASs based on whole blood samples

\begin{tabular}{|c|c|c|c|c|c|c|c|c|c|c|c|c|}
\hline \multirow[t]{3}{*}{ First author (year) } & \multirow[t]{3}{*}{ Country } & \multicolumn{3}{|c|}{ Discovery sample } & \multicolumn{3}{|c|}{ Validation sample } & \multirow[t]{3}{*}{$\operatorname{LDA}^{\mathrm{a}}$} & \multirow[t]{3}{*}{ Measurement } & \multirow{3}{*}{$\begin{array}{l}\text { Correction for } \\
\text { multiple tests }^{\mathrm{b}}\end{array}$} & \multirow[t]{3}{*}{ Smoking indicators ${ }^{c}$} & \multirow{3}{*}{$\begin{array}{l}\text { Identified number } \\
\text { of CpGs (genes) }^{d}\end{array}$} \\
\hline & & Size & Male & Mean age & Size & Male & Mean age & & & & & \\
\hline & & $(n)$ & (\%) & (year) & $(n)$ & (\%) & (year) & & & & & \\
\hline Guida, (2015) [21] & UK & 745 & 0 & $N A^{e}$ & NA & NA & NA & Yes $^{f}$ & $450 \mathrm{~K}$ & FWER & Current/former/never smoker (TQ) & $461(352)$ \\
\hline Zaghlool, $(2015)^{\mathrm{h}}$ [22] & Qatar & 123 & 35.8 & 37.9 & - & - & - & Yes $^{f}$ & $450 \mathrm{~K}$ & FDR & Smoker/non-smoker & $8(4)$ \\
\hline Besingi, $(2014)^{\mathrm{h}}[16]$ & Sweden & 432 & 45.8 & 42.7 & - & - & - & Yes $^{\mathrm{f}}$ & $450 \mathrm{~K}$ & FDR & Smoker/ non-smoker & $95(66)$ \\
\hline Dogan Mv Fau (2014) [17] & USA & 111 & 0 & 48.4 & 62 & 0 & 49.0 & Yes $^{\mathrm{f}}$ & $450 \mathrm{~K}$ quantitative-PCR & FDR & Smoker/non-smoker (PY) & $910(625)$ \\
\hline Elliott, (2014) [18] & UK & 192 & 100 & 48.5 & NA & NA & NA & Yes $^{f}$ & $450 \mathrm{~K}$ & FWER & Current/former/never smoker (PY) & $29(15)$ \\
\hline Harlid, (2014) [19] & USA & 908 & 0 & 55.7 & $200 / 476^{j}$ & 0 & 53.3 & No & $27 / 450 K^{k}$ & FDR & $\begin{array}{l}\text { Current/former/never smoker } \\
\text { (PY, TQ) }\end{array}$ & $13(13)$ \\
\hline Tsaprouni, (2014) [20] & UK & 464 & 70.5 & 55.4 & 356 & 0 & 60.1 & Yes $^{f}$ & $450 \mathrm{~K}$ & FWER & Current/former/never smoker & $30(15)$ \\
\hline Shenker, (2013) [14] & UK & 184 & 0 & NA & 190 & 71.6 & NA & No & $450 \mathrm{~K} /$ Pyrosequencing & FDR & Current/former/non -smoker (CL) & $9(4)$ \\
\hline Sun, (2013) [15] & USA & 972 & 29.3 & 66.3 & 239 & 28.5 & 40.8 & Yes $^{\mathrm{f}}$ & $27 / 450 K^{k}$ & FDR & Current/former/non -smoker (PY) & $5(5)$ \\
\hline Philibert, (2013) [13] & USA & 107 & 100 & 22.0 & NA & NA & NA & No & $450 \mathrm{~K}$ & FDR & Smoker/non-smoker (PY, CL) & $3(1)$ \\
\hline Zeilinger, (2013) [6] & Germany & 1793 & 51.3 & 60.9 & 478 & 48.3 & 51.8 & Yes $^{g}$ & $450 \mathrm{~K}$ & FWER & $\begin{array}{l}\text { Regular/occasional/former/never } \\
\text { smoker (ND, TQ, PY) }\end{array}$ & $187(94)$ \\
\hline Philibert, (2012) [11] & USA & 399 & 45.4 & 19.3 & NA & NA & NA & No & $450 \mathrm{~K}$ & FDR & Smoker/ non-smoker (PY) & $1(1)$ \\
\hline Wan, (2012) [12] & USA & 1085 & 54.4 & 57.3 & 369 & 35.8 & 47.5 & No & 27 K/Pyrosequencing & FDR & Current/former/never smoker (TQ, PY) & $15(14)$ \\
\hline Breitling, (2011) [5] & Germany & 177 & 50.8 & 54.0 & 316 & 59.5 & 55.0 & No & 27 KMMALDI-TOF $^{\prime}$ & FWER & Heavy/never/former smoker & $1(1)$ \\
\hline
\end{tabular}

aLDA leukocyte distribution adjustment

${ }^{\mathrm{b}}$ FWER family-wise error rate, FDR false discovery rate

${ }^{C} P Y$ pack-year, $N D$ numbers of cigarettes daily, TQ time of quitting smoking, $C L$ serum cotinine levels

dPart of $C P G$ sites located in the intergenic regions without gene antotions

eNot applicable

fThe influence of leukocyte composition was adjusted using the algorithm described by Houseman et al. [25]

9The influence of leukocyte composition was adjusted by subtype cell count

${ }^{\mathrm{h}}$ This study used the data published by other studies for replicating their significant sites

'The study was applied in the lymphocyte samples specifically

Two-hundred samples were analyzed by $450 \mathrm{~K}$, and 476 samples were analyzed by pyrosequencing

${ }^{k}$ The Illumina 27 and $450 \mathrm{~K}$ were used for exploratory and validation analysis, respectively

'MALDI-TOF = Sequenom matrix-assisted laser desorption ionization time-of-flight mass spectrometry 
Table $\mathbf{2}$ Characteristics summary of GSMSs based on whole blood samples

\begin{tabular}{|c|c|c|c|c|c|c|c|c|}
\hline \multirow[t]{3}{*}{ First author (year) } & \multirow[t]{3}{*}{ Country } & \multicolumn{3}{|l|}{ Sample } & \multirow[t]{3}{*}{ Measurement ${ }^{a}$} & \multirow[t]{3}{*}{ Smoking indicators ${ }^{\mathrm{b}}$} & \multirow[t]{3}{*}{ Gene(s) } & \multirow[t]{3}{*}{ Main finding ${ }^{e}$} \\
\hline & & Size & Male & Age mean & & & & \\
\hline & & $(n)$ & $(\%)$ & (year) & & & & \\
\hline Zhang, (2014) [8] & Germany & 3588 & 44.4 & 61.7 & MALDI-TOF & Current/former/never smoker (PY, ND, TQ) & $F 2 R L 3$ & $\begin{array}{l}\text { F2RL } 3 \text { methylation strongly decreased with } \\
\text { current smoking intensity and with lifetime } \\
\text { cumulative smoking, and reached a plateau } \\
\text { at higher current and cumulative smoking } \\
\text { intensity. Methylation levels increased with } \\
\text { time since cessation, but the time for full } \\
\text { recovery was more than } 20 \text { years. }\end{array}$ \\
\hline Shenker, (2013) [7] & UK & $81 / 180^{c}$ & $81.5 / 0$ & $N R^{d}$ & BPP & Current/former/never smoker (SD, CL) & AHRR, 2q37, 6p21 & $\begin{array}{l}\text { Combining four gene loci of } A H R R, 2 q 37, \\
\text { and } 6 p 21 \text { into a single methylation index } \\
\text { provided high positive, predictive, and } \\
\text { sensitivity values for predicting former smoking } \\
\text { status in both test ( } n=81, A \cup C=0.82) \text { and } \\
\text { validation }(n=180, A \cup C=0.83 \text { ) sample sets. }\end{array}$ \\
\hline Breitling, (2012) [23] & Germany & 1100 & 66.6 & 58.0 & MALDI-TOF & $\begin{array}{l}\text { Continuing smoker/quit after acute } \\
\text { event/quit before acute event/never smoking }\end{array}$ & $F 2 R L 3$ & $\begin{array}{l}\text { F2RL3 were significantly associated with } \\
\text { smoking history of patients with } C H D \\
\text { (median methylation intensities at } C p G \_4 \text {, } \\
\text { continuing smoker }=0.53 \text {; quit after acute } \\
\text { event }=0.51 \text {; quit before acute event }=0.66 \text {; } \\
\text { never smoking }=0.74)(p<0.001) \text {. }\end{array}$ \\
\hline
\end{tabular}

aALDI-TOF Sequenom matrix-assisted laser desorption ionization time-of-flight mass spectrometry, BPP Bisulphite pyrosequencing

${ }^{\mathrm{b}} P Y$ pack-year, $S D$ smoking duration, $N D$ numbers of cigarettes daily, $T Q$ time of quitting smoking, $C L$ serum cotinine levels

'The exploratory and validation analysis included 81 and 180 participants, respectively

${ }^{\mathrm{d}}$ Not reported

${ }^{\mathrm{e}} A U C$ area under the curve, $C H D$ coronary heart disease 


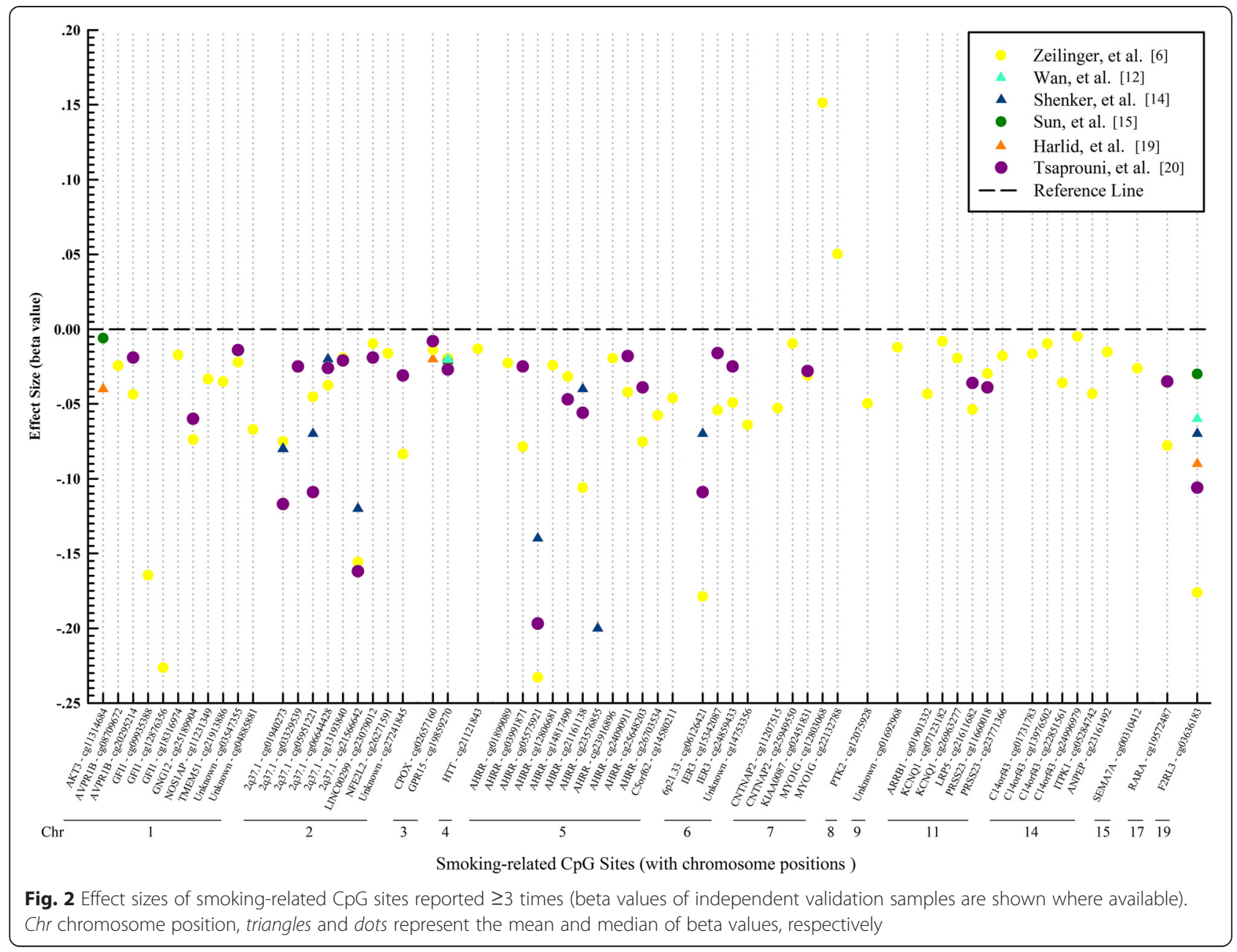

of smoking-associated changes after cessation. This finding was further confirmed in two studies of Tsaprouni et al. and Guida et al. [20, 21]. They described patterns consistent with the reversal of changes in methylation intensity after smoking cessation and suggested the existence of dynamic, reversible site-specific methylation alterations due to active smoking. In addition, a study investigating both tobacco and snuff smoking showed that tobacco smoking, not smokeless tobacco, was involved with DNA methylation, indicating that the majority of methylation changes might be caused by the burnt products of tobacco, not its basic components [16]. Lastly, the studies of Elliott et al. and Zaghlool et al. identified ethnic heterogeneity of smoking-associated differential methylation patterns in several loci $[18,22]$. For instance, smoking-associated differences of methylation at $\operatorname{cg} 05575921$ within $A H R R$ were significantly lower in individuals of European origin compared to South Asians [18]. The two studies speculated that either key aspects of smoking behavior had not been captured or there was a true ethnic difference in methylation response to smoking exposure.

\section{Study characteristics and results of GSMSs}

Four gene regions (F2RL3, AHRR, 2q37, and $6 p 21)$ and their related specific CpG sites, which had been identified by previous EWASs, were investigated in three whole blood-based GSMSs of active smoking (Table 2) [7, 8, 23]. All three studies were from Europe (Germany $=2$, UK $=1$ ) and population sizes varied from 261 to 3588. Two German studies evaluated the dose-response relationship between smoking exposure and methylation of F2RL3 by MALDI-TOF. The earlier one also revealed strong associations of smoking with adverse outcomes of coronary heart disease and concluded that methylation in F2RL3 might be a potential mediator of the detrimental impact of smoking [23]. The largest gene-specific study in 2014 was based on 3588 older adults from the general population [8]. This study disclosed clear dose-response relationships of F2RL3 methylation with both current and lifetime smoking intensity, as well as with time since smoking cessation. In particular, it was demonstrated that it took more than 20 years for a full "recovery" of methylation levels. The study by Shenker et al. assessed genome regions $A H R R, 2 q 37.1$, and $6 p 21.33$ via pyrosequencing [7]. 


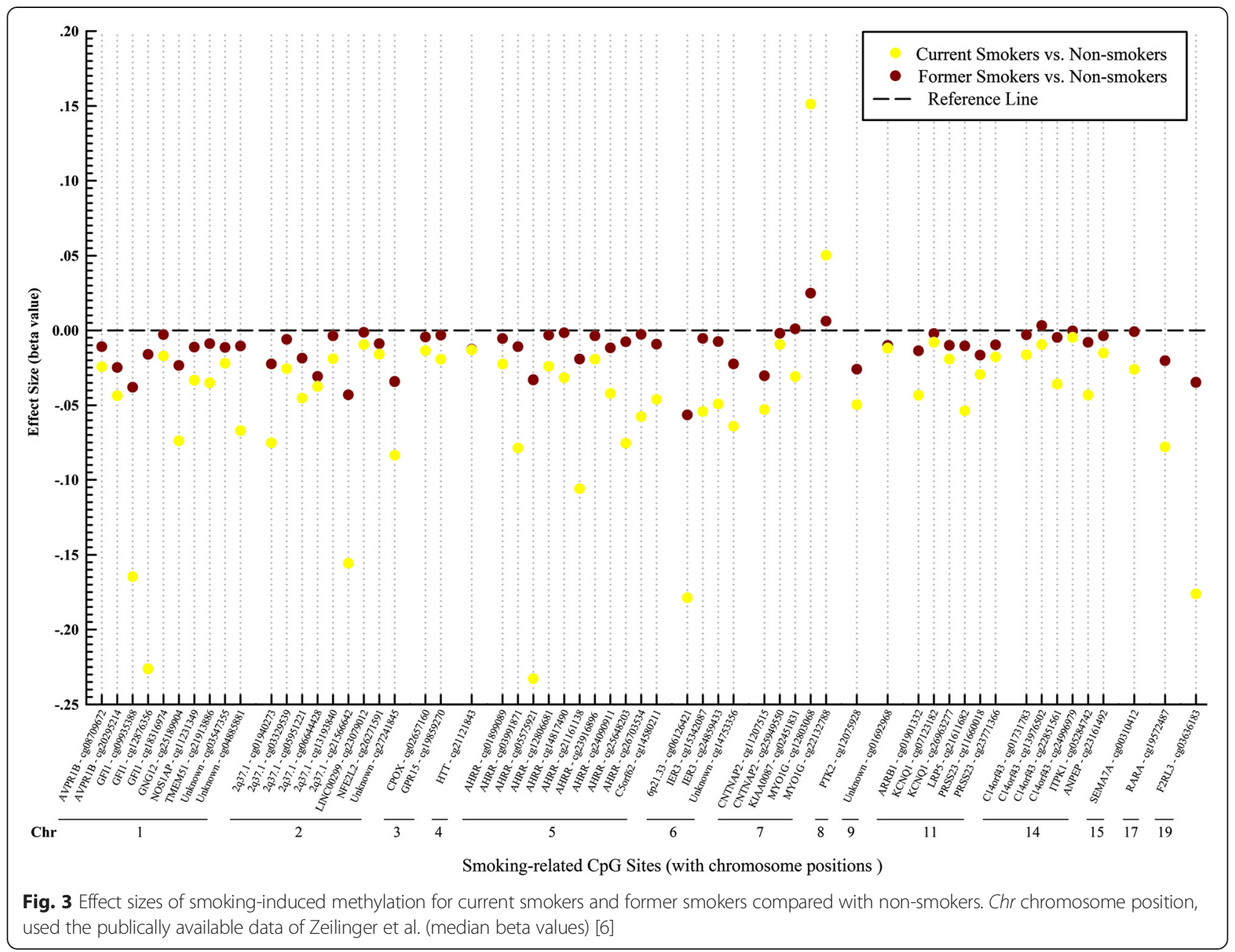

\section{Discussion}

Active smoking is an established critical factor for epigenetic modifications in blood DNA [4]. To our knowledge, this is the first systematic literature review on this topic. We identified 17 eligible articles, which explored the association of active smoking exposure with epigenetic changes in blood DNA. Overall, 1460 smoking-related CpG sites were identified in 14 EWASs, 62 of which were discovered by multiple $(\geq 3)$ studies. The most frequently reported sites were cg05575921 (AHRR), cg03636183 (F2RL3), cg1985 9270 (GPR15), and other loci within the intergenic regions $2 q 37.1$ and $6 p 21.33$. Prominent findings for these smoking-related genes were further analyzed in GSMSs to disclose dose-response relationships of smoking intensities and time since cessation with methylation levels. Taken together, these studies suggest the possibility of using methylation markers for a refined quantification of smoking exposure and to better predict the risks of smokingrelated diseases.

Smoking-induced methylation could occur in many regions of the human genome. In the annotated gene regions, approximately half of the smoking-related $\mathrm{CpG}$ sites are located in the body of specific genes (e.g., AHRR, F2RL3, etc.). Notably, their effect size was commonly higher than that of sites located at other parts, including the 1st exon, untranslated region (UTR), and transcription start site (TSS) (Additional file 1: Table S1, Fig. 2). Additionally, about one quarter of smoking-related loci are located in the intergenic regions, such as $2 q 37.1$ and $6 p 21.3$ (Additional file 1: Table S1).

For the sites located in the gene bodies, epidemiological studies have meanwhile observed biologically plausible associations with smoking-induced chronic diseases or cancers. For instance, the first discovered smokingassociated site cg03636183 is located in the body of gene F2RL3 (the coagulation factor II receptor-like 3 gene) [5], and was consistently confirmed in multiple EWASs and even replicated across racial groups $[15,17-19,22]$. The function of F2RL3 is coding the thrombin proteaseactivated receptor-4 (PAR-4), which is a protein expressed in various tissues over the body, including blood leukocytes and lung tissue, and plays a key role in platelet 
activation and cell signaling. This could partly explain why the methylation pattern of F2RL3 was found to be related to risks for cardiovascular diseases (CVD) and lung cancer, as well as to total mortality [26, 27]. Nevertheless, the role of F2RL3 as a potential mediator or just an indicator of smoking-related risk is still not well understood.

The strongest and most consistent associations have meanwhile been reported for CpG sites located in the body of $A H R R$, a well-known tumor suppressor. Smoking could trigger the generation of polycyclic aromatic hydrocarbons (PAHs) that affects the aryl hydrocarbon receptor (AHR), leading to alterations in the expression (and methylation status) of AHRR [3]. Thus, this gene could mediate detoxification of PAHs and might be involved in the metabolism of endogenous toxins from cigarette smoking [28]. A recent study by Zhang et al. disclosed clear dose-response relationships of AHRR methylation with both current and lifetime smoking exposure, as well as with smoking-related mortality outcomes [29]. Methylation at cg05575921in AHRR and a locus in 6p21.33 were additionally suggested to be promising candidates for enhancing cardiovascular risk prediction [29].

Irrespective of full understanding of the pathophysiological mechanisms, strong and consistent associations with smoking of a variety of CpG sites suggest their potential use as main correlates for smoking exposure. Two GSMSs have demonstrated the potential of several sites within F2RL3 as promising biomarkers for both current and past smoking exposure [8, 23]. A recent study has identified that cg05575921 within AHRR was both sensitive and specific for current smoking in adults with an area under the curve (AUC) of 0.99 , and efforts evaluating methylation of cg05575921 as a biomarker to guide smoking cessation are ongoing [30]. Further studies on loci within more smoking-related genes are in need to explore precise dose-response relationships to describe smoking exposure globally and understand their molecular mechanisms comprehensively.

In addition to the critical sites in gene bodies, there are several smoking-related sites within other genome regions, such as cg19859270, which are located at the 1stExon of gene GPR15 (G-protein-coupled receptor $15)$, and several loci in the intergenic region 2q37.1. These sites might make additional contributions to smoking exposure evaluation through a smoking-related methylation signature. Along with the significant loci in $A H R R$ and F2RL3, they could facilitate the construction of a quantitative approach with better specificity to differentiate never smokers from former smokers (validated $\mathrm{AUC}=0.83$, positive predictive value $=0.85$ ) [7]. In 2012, GPR15 was first reported in the study of Wan et al, along with its relationships with current and long-term smoking [12]. Afterwards, the study of Tsaprouni et al. presented that this gene was the only one showing a clear trend of increased gene expression in smokers compared to non-smokers with a prominent negative correlation between gene expression and methylation [20]. The author thus presumed that the reduction of methylation levels of locus cg19859270 within GPR15 in smokers would lead to increased transcription. This differential expression of GPR15 in smokers compared with never smokers was further confirmed by recent studies of Bauer et al. and Kõks et al. [31, 32]. In addition, as an HIV co-receptor, this gene was recently reported to interact with the ethnicity-dependent differential prevalence of HIV, especially HIV2 in African Americans [33]. Moreover, the six significant sites in locus $2 q 37.1$ are directly located adjacent to a cluster of alkaline phosphatase genes [34].

In the implementation and interpretation of EWASs and GSMSs based on blood samples, a potential limitation deserving particular attention is that whole blood DNA represents a mixture of DNA from various types of leucocytes that show partly different methylation patterns. Hence, smoking-related differential methylation may, in theory, partly reflect smoking-related shifts in leucocyte distribution. The majority of EWASs adjusted their analysis for leucocyte distribution with the algorithm of Houseman et al. [25]. However, although smoking is known to increase the overall numbers of leucocytes [35], the impact on leucocyte distribution still remains unclear [15-17, 20, 21]. Recently, GPR15 methylation has been shown to be linked with chronic inflammation via regulating $\mathrm{T}$ cell migration [32], which raised the possibility that for some loci, like GPR15, differential methylation might reflect a shift in blood cell mixture. On the other hand, a recent study compared the smoking-related methylation profiles in both buccal and whole blood samples and found that effect sizes in blood samples were similar to that in buccal samples [36]. This suggests that cell type distribution has no major impact for the majority of smoking-related differential methylation. Nevertheless, even if smoking-related methylation patterns were partly due to confounding by leucocyte distribution, they might still be useful as biomarkers for smoking exposure.

Our review was limited to smoking-associated DNA methylation changes among adults. Similar to the findings in adults, EWASs investigating the role of maternal smoking in newborns also identified differentially methylated $\mathrm{CpG}$ sites in several smoking-related genes, such as AHRR, MYO1G, and GFI1, but with less pronounced effect sizes. Interestingly, several loci, such as cg23067299 (AHRR) and cg05549655 (CYP1A1), were, so far, only discovered in studies assessing the impact of maternal smoking in newborns, whereas none of these studies reported differential methylation of $\operatorname{cg} 03636183$ (F2RL3) and cg19859270 (GPR15), two critical loci associated with 
adult smoking. These discrepancies are likely to be explained by the differences of exposure pathways and population susceptibilities [3,37], but more and larger EWASs in the respective age groups are needed to further clarify similarities and differences.

The number of known smoking-related CpG sites continues to increase. Given that smoking is an established risk factor for many chronic diseases, these loci could have important applications as objective biomarkers of both current and lifetime smoking exposure and for quantifying risks of smoking-related diseases. Recent GSMSs have already demonstrated strong dose-response relationships between methylation signatures and current and lifetime smoking exposure, as well as time since cessation of smoking [7, 8]. Furthermore, strong associations have been demonstrated between methylation signatures and a variety of major disease endpoints, including coronary artery diseases, lung cancer, and asthma [29, 38-41]. With further refinement of methylation signatures and further evaluation of the predictive value for these and additional disease outcomes, smoking-related methylation signatures might become a valuable tool for enhanced risk stratification and risk-adopted screening and treatment decisions in clinical practice. As a promising example, Teschendorff et al. constructed a smoking index score and subsequently showed that it was able to discriminate normal tissue from cancer tissue rather well [36], thereby demonstrating that smoking-related methylation indices could be useful risk indicators of smoking-induced health disorders.

This review has specific strengths and limitations. Strengths include the comprehensive search in two main databases, as well as strict adherence to standards of study selection, classification, and reporting. However, despite this comprehensive search strategy, we cannot exclude the possibility of having missed relevant studies, especially studies reported in languages other than English, or without full-length reports. Second, in most studies, smoking exposure was exclusively ascertained by self-reporting which is known to be less than perfect and most likely led to underestimation of true effects. Finally, our review was restricted to methylation patterns in blood DNA associated with active smoking. The focus on this sample matrix was a conscious decision due to its special relevance and ubiquitous availability in large epidemiological as well as routine point-of-care settings. Future research should address specific smoking-associated methylation signatures in various types of tissues (e.g., tumor samples, buccal cells). Also, apart from active smoking, methylation signatures reflecting passive smoking would be of major interest.

\section{Conclusions}

In summary, since the first discovery of smoking-related differential DNA methylation by Breitling et al. in 2011
[5], methylation of a large number of CpG sites has been consistently shown to be affected by smoking exposure. With few exceptions, active smoking was associated with reduced methylation levels, and smoking-related changes in methylation turned out to be reversible, with intermediate methylation levels among former smokers compared to current and never smokers. However, several studies have shown that it may take up to 20 years to reach a full "methylation recovery" $[8,21]$. Even though whole blood DNA presents a mixture of leucocytes subtypes, methylation of DNA from whole blood samples seems to be a powerful and highly informative biomarker not only for current smoking but also for lifetime history of smoking. Preliminary data suggest that smoking-related methylation signatures may also be very useful predictors of smoking-associated risks. Further research should aim for refinement of smoking-related methylation signatures and for their comprehensive validation with respect to a variety of disease outcomes in large epidemiological studies in order to best define the use of such signatures for research and clinical practice.

\section{Additional file}

Additional file 1: Table S1. Details of CpG sites published in all active smoking EWASs included in the review. This table includes 5 sheets: 1) the general information of reported $\mathrm{CpG}$ sites in each included manuscript; 2) CpG sites reported $\geq 2$ times; 3 ) characteristics of multiple reported ( $\geq 3$ times) CpG sites; 4 ) the list of active smoking-related CpG sites (no duplicates); and 5) data of Fig. 2. (XLSX 284 kb)

\section{Competing interests}

The authors declare that they have no competing interests.

\section{Authors' contributions}

All authors contributed to the drafting and critical revision of the manuscript, and all authors read and approved the final manuscript.

\section{Acknowledgements}

XG and MJ have been supported by the grant from the China Scholarship Council (CSC).

\section{Author details}

'Division of Clinical Epidemiology and Aging Research, German Cancer Research Center (DKFZ), Im Neuenheimer Feld 581, D-69120 Heidelberg, Germany. ${ }^{2}$ Division of Preventive Oncology, German Cancer Research Center (DKFZ) and National Center for Tumor Diseases (NCT), Im Neuenheimer Feld 460, D-69120 Heidelberg, Germany. ${ }^{3}$ German Cancer Consortium (DKTK), German Cancer Research Center (DKFZ), Im Neuenheimer Feld 280, D-69120 Heidelberg, Germany.

Received: 28 August 2015 Accepted: 6 October 2015

Published online: 16 October 2015

\section{References}

1. Mathers CD, Loncar D. Projections of global mortality and burden of disease from 2002 to 2030. PLoS Med. 2006;3(11), e442. doi:10.1371/journal.pmed.0030442.

2. Bakulski KM, Fallin MD. Epigenetic epidemiology: promises for public health research. Environ Mol Mutagen. 2014;55(3):171-83. doi:10.1002/em.21850.

3. Lee KW, Pausova Z. Cigarette smoking and DNA methylation. Front Genet. 2013:4:132. doi:10.3389/fgene.2013.00132. 
4. Philibert RA, Beach SR, Brody GH. The DNA methylation signature of smoking: an archetype for the identification of biomarkers for behavioral illness. Nebr Symp Motiv. 2014;61:109-27.

5. Breitling LP, Yang R, Korn B, Burwinkel B, Brenner H. Tobacco-smokingrelated differential DNA methylation: $27 \mathrm{~K}$ discovery and replication. Am J Hum Genet. 2011;88(4):450-7. doi:10.1016/j.ajhg.2011.03.003.

6. Zeilinger S, Kuhnel B, Klopp N, Baurecht H, Kleinschmidt A, Gieger C, et al. Tobacco smoking leads to extensive genome-wide changes in DNA methylation. PLoS One. 2013;8(5):e63812. doi:10.1371/journal.pone.0063812.

7. Shenker NS, Ueland PM, Polidoro S, van Veldhoven K, Ricceri F, Brown R, et al. DNA methylation as a long-term biomarker of exposure to tobacco smoke. Epidemiology. 2013;24(5):712-6. doi:10.1097/EDE.0b013e31829d5cb3.

8. Zhang Y, Yang R, Burwinkel B, Breitling LP, Brenner H. F2RL3 methylation as a biomarker of current and lifetime smoking exposures. Environ Health Perspect. 2014;122(2):131-7. doi:10.1289/ehp.1306937.

9. Novakovic B, Ryan J, Pereira N, Boughton B, Craig JM, Saffery R. Postnatal stability, tissue, and time specific effects of AHRR methylation change in response to maternal smoking in pregnancy. Epigenetics. 2014;9(3):377-86. doi:10.4161/epi.27248.

10. Panic N, Leoncini E, de Belvis G, Ricciardi W, Boccia S. Evaluation of the endorsement of the preferred reporting items for systematic reviews and meta-analysis (PRISMA) statement on the quality of published systematic review and meta-analyses. PLoS One. 2013;8(12):e83138. doi:10.1371/journal.pone.0083138.

11. Philibert RA, Beach SR, Brody GH. Demethylation of the aryl hydrocarbon receptor repressor as a biomarker for nascent smokers. Epigenetics. 2012;7(11):1331-8. doi:10.4161/epi.22520.

12. Wan ES, Qiu W, Baccarelli A, Carey VJ, Bacherman H, Rennard SI, et al. Cigarette smoking behaviors and time since quitting are associated with differential DNA methylation across the human genome. Hum Mol Genet. 2012;21(13):3073-82. doi:10.1093/hmg/dds135.

13. Philibert RA, Beach SR, Lei MK, Brody GH. Changes in DNA methylation at the aryl hydrocarbon receptor repressor may be a new biomarker for smoking. Clin Epigenetics. 2013;5(1):19. doi:10.1186/1868-7083-5-19.

14. Shenker NS, Polidoro S, van Veldhoven K, Sacerdote C, Ricceri F, Birrell MA, et al. Epigenome-wide association study in the european prospective investigation into cancer and nutrition (EPIC-Turin) identifies novel genetic loci associated with smoking. Hum Mol Genet. 2013;22(5):843-51. doi:10.1093/hmg/dds488.

15. Sun YV, Smith AK, Conneely KN, Chang Q, Li W, Lazarus A, et al. Epigenomic association analysis identifies smoking-related DNA methylation sites in African Americans. Hum Genet. 2013;132(9):1027-37. doi:10.1007/s00439-013-1311-6.

16. Besingi W, Johansson A. Smoke-related DNA methylation changes in the etiology of human disease. Hum Mol Genet. 2014;23(9):2290-7. doi:10.1093/hmg/ddt621.

17. Dogan MV, Shields B, Cutrona C, Gao L, Gibbons FX, Simons R et al. The effect of smoking on DNA methylation of peripheral blood mononuclear cells from African American women. BMC Genomics. 2014;15(1471-2164 (Electronic)):151. doi:10.1186/1471-2164-15-151.

18. Elliott HR, Tillin T, McArdle WL, Ho K, Duggirala A, Frayling TM, et al. Differences in smoking associated DNA methylation patterns in South Asians and Europeans. Clin Epigenetics. 2014;6(1):4. doi:10.1186/1868-7083-6-4.

19. Harlid S, Xu Z, Panduri V, Sandler DP, Taylor JA. CpG sites associated with cigarette smoking: analysis of epigenome-wide data from the sister study. Environ Health Perspect. 2014;122(7):673-8. doi:10.1289/ehp.1307480.

20. Tsaprouni LG, Yang TP, Bell J, Dick KJ, Kanoni S, Nisbet J, et al. Cigarette smoking reduces DNA methylation levels at multiple genomic loci but the effect is partially reversible upon cessation. Epigenetics. 2014;9(10):1382-96. doi:10.4161/15592294.2014.969637.

21. Guida F, Sandanger TM, Castagne R, Campanella G, Polidoro S, Palli D, et al. Dynamics of smoking-induced genome-wide methylation changes with time since smoking cessation. Hum Mol Genet. 2015;24(8):2349-59. doi:10.1093/hmg/ddu751.

22. Zaghlool SB, Al-Shafai M, Al Muftah WA, Kumar P, Falchi M, Suhre K. Association of DNA methylation with age, gender, and smoking in an Arab population. Clin Epigenetics. 2015;7(1):6. doi:10.1186/s13148-014-0040-6.

23. Breitling LP, Salzmann K, Rothenbacher D, Burwinkel B, Brenner H. Smoking, F2RL3 methylation, and prognosis in stable coronary heart disease. Eur Heart J. 2012;33(22):2841-8. doi:10.1093/eurheartj/ehs091.

24. Lohmueller KE, Pearce CL, Pike M, Lander ES, Hirschhorn JN. Meta-analysis of genetic association studies supports a contribution of common variants to susceptibility to common disease. Nat Genet. 2003;33(2):177-82. doi:10.1038/ng1071.
25. Houseman EA, Accomando WP, Koestler DC, Christensen BC, Marsit CJ, Nelson $\mathrm{HH}$, et al. DNA methylation arrays as surrogate measures of cell mixture distribution. BMC Bioinformatics. 2012;13:86. doi:10.1186/1471-2105-13-86.

26. Zhang Y, Schottker B, Ordonez-Mena J, Holleczek B, Yang R, Burwinkel B, et al. F2RL3 methylation, lung cancer incidence and mortality. Int J Cancer. 2015;137(7):1739-48. doi:10.1002/ijc.29537.

27. Zhang Y, Yang R, Burwinkel B, Breitling LP, Holleczek B, Schottker B, et al. F2RL3 methylation in blood DNA is a strong predictor of mortality. Int J Epidemiol. 2014;43(4):1215-25. doi:10.1093/ije/dyu006.

28. Watanabe T, Imoto I, Kosugi Y, Fukuda Y, Mimura J, Fujii Y, et al. Human arylhydrocarbon receptor repressor (AHRR) gene: genomic structure and analysis of polymorphism in endometriosis. J Hum Genet. 2001;46(6):342-6. doi:10.1007/s100380170070.

29. Zhang Y, Schottker B, Florath I, Stock C, Butterbach K, Holleczek B et al. Smoking-Associated DNA Methylation Biomarkers and Their Predictive Value for All-Cause and Cardiovascular Mortality. Environ Health Perspect. 2015 doi:10.1289/ehp.1409020.

30. Philibert R, Hollenbeck N, Andersen E, Osborn T, Gerrard M, Gibbons FX, et al. A quantitative epigenetic approach for the assessment of cigarette consumption. Front Psychol. 2015;6:656. doi:10.3389/fpsyg.2015.00656.

31. Bauer M, Linsel G, Fink B, Offenberg K, Hahn AM, Sack U, et al. A varying T cell subtype explains apparent tobacco smoking induced single CpG hypomethylation in whole blood. Clin Epigenetics. 2015;7(1):81. doi:10.1186/s13148-015-0113-1.

32. Koks G, Uudelepp ML, Limbach M, Peterson P, Reimann E, Koks S. SmokingInduced Expression of the GPR15 Gene Indicates Its Potential Role in the Chronic Inflammatory Pathologies. Am J Pathol. 2015. doi:10.1016/j.ajpath.2015.07.006.

33. Dogan MV, Xiang J, Beach S, Cutrona C, Gibbons F, Simons R et al. Ethnic and smoking associated DNA methylation changes at HIV co-receptor GPR15. Frontiers in Psychiatry. 2015;6. doi:10.3389/fpsyt.2015.00132.

34. Dua P, Kang HS, Hong SM, Tsao MS, Kim S, Lee DK. Alkaline phosphatase $A L P P L-2$ is a novel pancreatic carcinoma-associated protein. Cancer Res. 2013;73(6):1934-45. doi:10.1158/0008-5472.CAN-12-3682.

35. Schwartz J, Weiss ST. Cigarette smoking and peripheral blood leukocyte differentials. Ann Epidemiol. 1994;4(3):236-42.

36. Teschendorff AE, Yang Z, Wong A, Pipinikas CP, Jiao Y, Jones A et al. Correlation of smoking-associated DNA methylation changes in buccal cells with DNA methylation changes in epithelial cancer. JAMA Oncol. 2015;1(4):476-85. doi:10.1001/jamaoncol.2015.1053.

37. Markunas CA, Xu Z, Harlid S, Wade PA, Lie RT, Taylor JA et al. Identification of DNA Methylation Changes in Newborns Related to Maternal Smoking during Pregnancy. Environ Health Perspect. 2014. doi:10.1289/ehp.1307892.

38. Zhang Y, Schottker B, Ordonez-Mena J, Holleczek B, Yang R, Burwinkel B et al. F2RL3 methylation, lung cancer incidence and mortality. Int J Cancer. 2015. doi:10.1002/ijc.29537.

39. Zong DD, Ouyang RY, Chen P. Epigenetic mechanisms in chronic obstructive pulmonary disease. Eur Rev Med Pharmacol Sci. 2015;19(5):844-56.

40. Lovinsky-Desir S, Miller RL. Epigenetics, asthma, and allergic diseases: a review of the latest advancements. Curr Allergy Asthma Rep. 2012;12(3):211-20. doi:10.1007/s11882-012-0257-4.

41. Steenaard RV, Ligthart S, Stolk L, Peters MJ, van Meurs JB, Uitterlinden AG, et al. Tobacco smoking is associated with methylation of genes related to coronary artery disease. Clin Epigenetics. 2015;7(1):54. doi:10.1186/s13148-015-0088-y.

\section{Submit your next manuscript to BioMed Central and take full advantage of:}

- Convenient online submission

- Thorough peer review

- No space constraints or color figure charges

- Immediate publication on acceptance

- Inclusion in PubMed, CAS, Scopus and Google Scholar

- Research which is freely available for redistribution 